\title{
Emergency treatment principles in intracranial hypertension
}

\author{
St.M. Iencean ${ }^{1}$, A.St. Iencean ${ }^{2}$, I. Poeata ${ }^{1}$ \\ 1"Grigore T. Popa" University of Medicine and Pharmacy Iasi \\ ${ }^{2}$ Emergency Hospital "N. Oblu” Iasi
}

As general principles the treatment of intracranial hypertension depends on the type of intracranial hypertension and on the stage of the illness. First the treatment is an etiologic one in order to remove the cause that has caused the intracranial pressure increase: intracranial masses producing elevated ICP should be removed when possible. Simultaneously, there is an attempt to stop the pathogenic mechanisms that impact on the nervous structures, and a symptomatic treatment is applied in order to reduce the intensity of the clinical syndrome.

In the case of a patient with a suspicion of decompensating intracranial hypertension, one must perform and assess:

- the complete evaluation of the patient with the acute ICH syndrome: one must assess the consciousness level, the conscience state, the aspect of the pupils must be monitored and the Glasgow score must be established,

- the vital conditions: free aerial ways, respiration and the circulatory function; resuscitation operations are performed if needed, with oro-tracheal intubation, etc. In case of traumatisms, a cervical vertebralmedullar lesion must be excluded, and possible lesions with a vital risk must be revealed if they require an immediate solution.

- the emergency exploration by a cranial-cerebral computer tomography establishes the diagnosis and the therapeutic option is decided. $(2,8,12,14,16,30)$

Thus the first therapeutic actions are determined depending on the lesional characteristics:

- the expansive intracranial process (tumor, traumatic process), which has induced the ICH syndrome is surgically extirpated in order to remove the supplementary pathogenic volume with a compressive effect (mass effect), and which leads to a brain edema.

- the expansive intracranial process that blocks the CSF circulation paths with the production of an obstructive hydrocephalus and the ICH syndrome requires at first the performance of a ventricular drainage and then, based on the characteristics of the lesion, there will be an intervention for its extirpation.

- in the blood blockage of the ventricular system in intracranial hemorrhages, with the occurrence of the ICH syndrome due to obstructive hydrocephalus, the ventricular drainage is performed, and then the exploration and the treatment of the intracranial hemorrhage.

- the presence of a brain edema without any other lesion requires the antiedematous treatment in order to reduce the intracranial pressure, at the same time as maintaining the a cerebral perfusion pressure within normal limits by the 
administration of mannitol, furosemide, dexamethasone, vasopressors, etc.

- in non-surgical multiple traumatic lesions, a pathogenic therapy is applied in order to decrease the intracranial pressure and stop the evolution of secondary traumatic lesions.

- an appropriate cerebral perfusion pressure must be maintained in order to prevent the cerebral ischemia. PCP must be higher than $60-80 \mathrm{~mm} \mathrm{Hg}$.

- avoidance of the situations when the cerebral metabolism increases:

- avoidance of stimuli: aspiration strictly when needed, patient's transportation, etc.

- hyperthermia must be vigorously discouraged: antithermic medication, muscular relaxing substances, cold packing up; the moderate hypothermia is an efficient procedure to decrease the cerebral metabolism and for cerebral protection.

- the evaluation of the biological parameters with the urgent control of the sanguine glycemia; in the case of the neurosurgical patient with a normal carbohydrate metabolism, it is not recommended to use glucose solutions as this may worsen the brain edema or the cerebral ischemic lesions

- the decrease in the arterial and intracranial venous sanguine volume by:

- head raising, which facilitates the venous drainage and increases the CSF removal

- the hyperventilation decreases PCO2 leading to an arterial vasoconstriction that decreases the cerebral sanguine volume

- AT monitoring, in order to hinder AT variations: correction of the arterial hypotension (decrease in the cerebral sanguine perfusion), as well as of the high blood pressure(increase in the intracranial pressure)
- pharmacologic methods to decrease the metabolism and the $\mathrm{O} 2$ needs with the reduction of the cerebral sanguine volume (thiopental 4-6 $\mathrm{mg} / \mathrm{kg}$ or propofol 2-3 $\mathrm{mg} / \mathrm{kg}$ ), but with the cerebral perfusion pressure maintained within normal limits

- the signs of ICH decompensation unilateral mydriasis - (the cerebral compliance has been exceeded) require urgent measures of ICP decrease and in order to prevent a further ICP increase:

- the occurrence of acute hydrocephalus (by any mechanism) imposes the CSF drainage with a ventricular catheter, which rapidly decreases the intracranial pressure,

- in order to prevent the cerebral herniation, a hemi-craniectomy can be used with a partial resection of the temporal lobe,

- in cases of malign brain edema, which is resistant to medicine therapies, a hemicraniectomy can be performed with the removal of the cranial bone and the sectioning of the dura mater in order to allow the expansion of the cerebral parenchyma during the acute stage,

- in brainstem compressions, at the same time as the aggressive medication treatments, the decompression of the posterior cerebral fosse is performed by means of a sub-occipital craniectomy, completed by a ventricular drainage if there is also hydrocephalus.

- in the case of an operated patient, with a post-surgery ICP monitoring, the normalization of the intracranial pressure values is monitored, as well as the improvement of the cerebral circulation:

- if the cerebral perfusion pressure is below $70 \mathrm{~mm} \mathrm{Hg}$, the correction treatment of the intracranial sanguine contribution is started, even if the ICP is still high, by means of the administration of colloidal 
solutions, intravenous vasopressors, etc.

- if the intracranial pressure has values of less than $20 \mathrm{~mm} \mathrm{Hg}$ and the cerebral perfusion pressure is above $70 \mathrm{~mm} \mathrm{Hg}$, the patient is supervised, and the evolution of the ICP and PCP values are monitored.

- if the intracranial pressure is higher than $20 \mathrm{~mm} \mathrm{Hg}$, the agitated patient is sedated and the permeability of the respiratory paths is checked; the head is raised at 25 - 30 degrees from horizontal, which leads to a moderate ICP decrease through an increase in the venous drainage. The ICP normalization during these maneuvers does not require other therapeutic actions regarding the intracranial pressure.

- in the case of the patient who has not been in a surgery, but who has an ICP monitoring indication, one must survey and correct the intracranial pressure increases and the cerebral perfusion pressure decreases. The ICP values of more than 20 $\mathrm{mm} \mathrm{Hg}$ require the agitated patient's sedation and the check of the respiratory path permeability. The decreased value of the cerebral perfusion pressure requires the specific therapy for the maintenance of the cerebral circulation within normal limits.

Treatment scheme in traumatic acute intracranial hypertension

The therapeutic stages for patients with traumatic brain injury and an acute $\mathrm{ICH}$ syndrome aim to reduce the ICP increases and to maintain the PCP within normal limits. The treatment is performed progressively, depending on the monitoring values of ICP, PCP and of the other parameters. $(2,7,8,12,14)$

- sedation and perhaps a moderate hyperventilation; this is applied as long as ICP $<20 \mathrm{~mm} \mathrm{Hg}$ for the first 12 hours:

- sedatives
- $\mathrm{PaCO} 2$ is maintained at $\approx 35 \mathrm{~mm} \mathrm{Hg}$ in the case of mechanically ventilated patients

- when the ICP value is above $20 \mathrm{~mm}$ $\mathrm{Hg}$, one must verify whether the increased pressure values are caused by the agitated state or if there is a mechanical obstruction that may cause the pressure increase. The patient who manifests a psycho-motor agitation is sedated, and, if ICP decreases below $20 \mathrm{~mm} \mathrm{Hg}$, the monitoring, as well as the pathogenic and symptomatic therapy in the intensive care ward are continued.

- CSF drainage with the ventricular catheter: if ICP $>20 \mathrm{~mm} \mathrm{Hg}$ for more than 5 minutes, CSF drainage is used whenever needed, as long as the operation proves to be efficient.

- use of diuretics: if ICP is maintained high above $20 \mathrm{~mm} \mathrm{Hg}$ for more than 5 minutes, the following procedures are used:

- osmotic diuresis: mannitol administration.

- ansa diuretics: furosemide is used

The combination of these two diuretics is more efficient and is performed while monitoring of osmolarity and of the sanguine electrolytes.

- hyperventilation with the maintenance of $\mathrm{PaCO} 2$ at values of $25-30 \mathrm{~mm} \mathrm{Hg}$. Hyperventilation causes cerebral vasoconstriction and the ICP decrease by decreasing the cerebral sanguine flux. Hyperventilation is applied intermittently and it is recommended on the second day after a severe cranial-cerebral traumatism.

- hypertensive therapy for the increase in the systemic blood pressure, which can ensure a normal cerebral perfusion pressure if the intracranial pressure is increased. If cerebral contusion lesions are evident (areas of cerebral hemorrhage - hemorrhagic contusion), the systemic arterial pressure 
can increase up to $150-170 \mathrm{~mm} \mathrm{Hg}$; if there are no areas of cerebral contusion, the blood pressure can increase to $180 \mathrm{~mm} \mathrm{Hg}$.

- controlled hypothermia, maintaining an approximate temperature of $35^{\circ} \mathrm{C}$.

- administration of a hypertonic $\mathrm{NaCl}$ solution with a concentration of $7.5 \%$.

- surgical decompression: if the cerebral CT exploration shows that the cerebral edema is very important without revealing a compressive intracranial lesion, performing a large decompressive craniectomy, unilateral or bilateral, must be considered.

- the anesthetic administration is performed with an electro-encephalic monitoring up to doses that diminish the EEG activity, using:

- non-barbituric hypnotic substances: etomidate, propofol.

- barbituric substances: phenobarbital, thiopental (barbituric coma).

- Trometamol administration (THAM), which generates a partial decrease in $\mathrm{CO} 2$ pressure and produces a cerebral vasoconstriction. The condition is for the reactivity of the cerebral vessels to $\mathrm{CO} 2$ to be intact

- the lumbar puncture of CSF drainage is applied after the exhaustion of the other therapeutic means. The cerebral computer tomography exploration must reveal the presence of the basal cisterns and of the lateral ventricles. The risk of inducing a cerebral hernia by this operation, which is not recommended in intracranial hypertension, is considered to be smaller than the unfavorable evolution by the accentuation of the intracranial pressure increase. $(12,31)$

Treatment scheme in intracranial hypertension of ischemic stroke
The emergency treatment of the massive ischemic cerebral or cerebellar stroke, which may lead to an ICH syndrome, includes:

- securing the vital conditions: free aerial ways, respiration and circulatory function, in the circumstances of a critical condition or of a coma,

- the intravenous administration of a recombined activator of tissue plasminogen (rTPA, rtPA) during the first three hours from the beginning of the stroke in a dose of $0.9 \mathrm{mg} / \mathrm{kg}$; maximum $90 \mathrm{mg}$. (1, 13, 17, $18,24)$

The intravenous administration of streptokinase or of other thrombolytic agents does not have the same effects as the rtPA administration.

- if it is necessary, a progressive decrease in the systemic blood pressure is performed,

- administration of osmotic diuretics (mannitol) if there are any signs of intracranial hypertension decompensation,

- hyperventilation when intracranial hypertension decompensation and cerebra hernia occurrence are imminent,

- cortico-therapy is not recommended in the treatment of the cerebral edema in cerebral ischemic stroke

- surgical intervention if the ICH decompensation occurs:

- decompression and the evacuation of a cerebellar stroke with a compressive effect on the brainstem, perhaps a ventricular drainage too,

- decompression and the evacuation of a massive cerebral hemispheric stroke, which can reduce the intracranial hypertension, but the surviving patients are left with major neurologic deficits. 
Corresponding author:

A.St. Iencean

Emergency Hospital "N. Oblu” Iasi

andrei_steffan@yahoo.com

\section{References}

1. Adelson PD, Bratton SL, Carney NA, Chesnut RM, du Coudray HE, et al The use of barbiturates in the control of intracranial hypertension in severe pediatric traumatic brain injury. Pediatr Crit Care Med. 2003; 4 : S49-52.

2. Adelson PD, Bratton SL, Carney NA, Chesnut RM, et al The role of cerebrospinal fluid drainage in the treatment of severe pediatric traumatic brain injury. Pediatr Crit Care Med. 2003, 4(3 Suppl): S38-9.

3. Adelson PD, Bratton SL, Carney NA, Chesnut RM, et al Use of hyperosmolar therapy in the management of severe pediatric traumatic brain injury. Pediatr Crit Care Med. 2003; 4(3 Suppl): S40-4.

4. Adelson PD, Bratton SL, Carney NA, Chesnut RM, et al Surgical treatment of pediatric intracranial hypertension. Pediatr Crit Care Med. 2003; 4(3 Suppl): S56-9.

5. Adelson PD, Bratton SL, Carney NA, Chesnut RM, et al The use of corticosteroids in the treatment of severe pediatric traumatic brain injury. Pediatr Crit Care Med. 2003; 4(3 Suppl): S60-4.

6. Adelson PD, Bratton SL, Carney NA, et al Critical pathway for the treatment of established intracranial hypertension in pediatric traumatic brain injury. Pediatr Crit Care Med. 2003; 4(3 Suppl): S65-7.

7. Allan R, Chaseling R. Subtemporal decompression for slit-ventricle syndrome: successful outcome after dramatic change in intracranial pressure wave morphology. Report of two cases. J Neurosurg. 2004; 101( Suppl): 214-7.

8. Battison C, Andrews PJ, Graham C, et al Randomized, controlled trial on the effect of a $20 \%$ mannitol solution and a $7.5 \%$ saline $/ 6 \%$ dextran solution on increased intracranial pressure after brain injury. Crit Care Med. 2005; 33(1): 196-202.

9. Berger S, Schwarz M, Huth R. Hypertonic saline solution and decompressive craniectomy for treatment of intracranial hypertension in pediatric severe traumatic brain injury. J Trauma. 2002 53(3): 558-63.

10. Coppage KH, Sibai BM. Treatment of hypertensive complications in pregnancy. Curr Pharm Des. 2005; 11(6): 749-57.

11. Cruz J, Minoja G, Okuchi K. Major clinical and physiological benefits of early high doses of mannitol for intraparenchymal temporal lobe hemorrhages with abnormal pupillary widening: a randomized trial. Neurosurgery. 2002, 51(3): 628-37.

12. Demchuk AM, Burgin WS, Christou I, et al. Thrombolysis in brain ischemia (TIBI) transcranial
Doppler flow grades predict clinical severity, early recovery, and mortality in patients treated with intravenous tissue plasminogen activator. Stroke. 2001; 32: 89-93.

13. Diener HC, Ringelstein EB, von Kummer R, et al. Treatment of acute ischemic stroke with the lowmolecular-weight heparin certoparin: results of the TOPAS trial: Therapy of Patients with Acute Stroke (TOPAS) Investigators. Stroke. 2001; 32: 22-29.

14. Donnan GA, Davis SM. Surgical decompression for malignant middle cerebral artery infarction: a challenge to conventional thinking. Stroke. 2003; 34(9): 2307.

15. El-Watidy S. Bifrontal decompressive craniotomy in a 6-month-old infant with posttraumatic refractory intracranial hypertension. Pediatr Neurosurg. 2005; 41(3): 151-4.

16. Figaji AA, Fieggen AG, Peter JC. Early decompressive craniotomy in children with severe traumatic brain injury.Childs Nerv Syst. 2003; 19(9): 666-73.

17. Fraser JF, Hartl R. Decompressive craniectomy as a therapeutic option in the treatment of hemispheric stroke. Curr Atheroscler Rep. 2005; 7(4): 296-304.

18. 18.Georgiadis AL, Suarez JI. Hypertonic saline for cerebral edema. Curr Neurol Neurosci Rep. 2003; 3(6): 524-30

19. Gupta R, Connolly ES, Mayer S, Elkind MS. Hemicraniectomy for massive middle cerebral artery territory infarction: a systematic review. Stroke. 2004; 35(2): 539-43

20. Iencean St M A new classification and a synergetical pattern in intracranial hypertension. Medical Hypotheses, 2002; 58(2): 159-63.

21. Iencean StM Brain edema - a new classification. Med Hypotheses. 2003; 61(1): 106-9.

22. Iencean StM, Ciurea AV Intractranial Hypertension, Nova Science Publishers, New York, 2009.

23. Kontopoulos V, Foroglou $\mathrm{N}$, Patsalas $\mathrm{J}$, et al Decompressive craniectomy for the management of patients with refractory hypertension: should it be reconsidered? Acta Neurochir (Wien). 2002; 144(8): 791-6.

24. Lee AG, Pless M, Falardeau J, et al The use of acetazolamide in idiopathic intracranial hypertension during pregnancy. Am J Ophthalmol. 2005; 139(5): 855-9.

25. Owler BK, Parker G, Halmagyi GM, Pseudotumor cerebri syndrome: venous sinus obstruction and its treatment with stent placement. J Neurosurg. 2003; 98(5): 1045-55.

26. Watling CJ, Cairncross JG. Acetazolamide therapy for symptomatic plateau waves in patients with brain tumors. Report of three cases. J Neurosurg. 2002; 97(1): 224-6. 